Send your research letters to the editor British Dental Journal, 64 Wimpole Street, LondonW1G8YSE-mail bdj@bda dentistry.org.uk

Priority will be given to letters less than 500 words long. Authors must sign the letter, which may be edited for reasons of space

\section{Scientific basis}

Sir, I write to respond to Dr Marks' letter (BDJ 2005, 198:351).We are indeed privileged to be working in a profession that can make a real difference to the quality of our patients' lives. Dr Marks' letter is an enlightening one and it is indeed his patient's good fortune that he recognised the importance of muscle dysfunction in chronic head, neck and facial pain.

Whilst it has been shown that contact of the anterior teeth (and perioral tissues) may 'shut down' strong muscle activity of masseter and temporalis ${ }^{1,2}$ the therapeutic goal of any treatment regimen is to return the body to an optimal (and stable) state of comfort and function. ${ }^{3}$ In this case harmony between muscles, joint and posture (as the teeth are but innocent bystanders).

The interested practitioner is encouraged to review the scientific and objective recording of centric relation (CR $)^{4,5}$ and also the importance of head posture. ${ }^{6}$ The jaw joint plays more of a supportive role usually $^{7-9}$ and internal derangements and their significance were being discussed in The Lancet 150 years ago. ${ }^{9}$

Dentistry owes a great debt to the pioneers of the mechanically based gnathological principles and theories. In this millennium we need to move on from this excellent historical foundation and I believe it is vital to pursue a 'scientific basis' to its approach via anatomy and physiology in order to serve our profession and patients best in the future. ${ }^{10}$

\section{S. Bray}

Poole

doi: 10.1038/sj.bdj.4812445

1. Hannam A G, Mathews B, Yemm R. Changes in the activity of the masseter muscle following tooth contact in man. Arch Oral Biol 1969: 14: 1401.

2. Schoenen J. Exteroceptive suppression of temporalis muscle activity: methodological and physiological aspects. Cephalalgia 1993; 13:3-10.

3. Dickerson W G, Chan CA, Mazzocco M W. Concepts of Occlusion. Signature 2004 (7).

4. Hickman D M, Cramer R. The effect of different condylar positions on masticatory muscle electromyographic activity in humans. Oral Surg Oral Med Oral Pathol Oral Radiol Endod. 1998; July; 86 (1): 18-23.

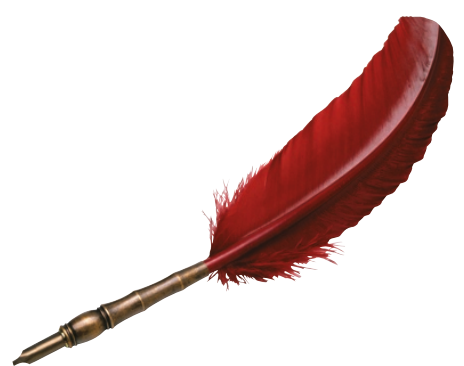

5. Hickman D M, Crmer R, Stauber W T, Arch Oral Biol 1993; Mar 38 (3):261

6. Kibana Y, Ismijima T, Hirai T, Occlusal support and head posture, J Oral Rehabil 2002; 29: 58-63.

7. Katzberg M D et al. Internal derangements and arthritis of the TMJ. Radiology 1983; 146: 107-112.

8. Katzberg M D et al. Internal derangements of the TMJ: Findings in the Paediatric Age Group. Radiology 1985; 154: 125-127.

9. Bray S J. The Strange Case of the Clicking Joint. Firsthand (FGDP UK) Journal. Apr 2004 : 4-5.

10. Cooper B. Neuromuscular Dentistry - The next millennium, Scientific Rationale for Biomedical Instrumentation, Anthology Volume V. Seattle, WA: ICCMO 1999

\section{Irradiated jaws}

Sir, I read with interest the article 'Antibiotic prophylaxis and third molar surgery' (BDJ 2005, 198:327) and agree completely with the authors opinion.

Nevertheless, there have been many controversial issues on dental extraction/minor oral surgery in irradiated jaws. One of which of course, is the use of antibiotic prophylaxis. In general, whenever we talk about patients with a history of radiotherapy, we usually refer to those who have direct radiation to the jawbones (normally for treatment oral cancer).

Hence that may explain why most maxillofacial surgeons will recommend prophylaxis use prior to extraction of lower posterior teeth (where the jawbone is dense) ${ }^{1}$. In my part of the world, besides oral cancer patients, we also see quite a large number of patients with a history of radiotherapy for the treatment of nasopharyngeal carcinoma (NPC), with effects to the oral cavity similar to that seen in oral cancer patients. Because of the difference in location of radiation, the recommendation for antibiotic use in this group of patients is different.

Thus, I would like to bring to your attention the protocol recommended by Tong et al. ${ }^{2}$ that is tailored specifically for NPC patients. This recommendation was formulated based on their experience with NPC patients in Hong Kong, where their study had shown that the risk of complicated wound healing and oral radio-necrosis after extraction could be explained by the radiation dose received by the bone and socket in which the teeth were situated. Extraction of teeth in areas outside the high radiation dose areas can be safely undertaken with antibiotic cover. Thus, they suggested that it would be safe to extract maxillary and mandibular anterior teeth if tooth extraction is considered absolutely necessary.

Tong et al. ${ }^{2}$ claimed that the extraction of premolars and mandibular molars is not associated with significant risk provided that an atraumatic technique and antibiotic cover are used. They think that antibiotic therapy is useful as an adjunctive treatment modality, thus it is a common practice to provide antibiotic cover for post-irradiation extractions. Among the antibiotics that could be prescribed is a single dose of either $3 g$ oral amoxicillin or in those patients allergic to penicillin, $600 \mathrm{mg}$ of oral clindamycin, one hour preoperatively.

Alternatively it has also been suggested that amoxycillin $250 \mathrm{mg}$ or metronidazole $200 \mathrm{mg}$ be given three times daily for 3-5 days of post-surgery to prevent infection in the healing phase. Problems arising from these extractions are manageable with relatively simple measures of wound debridement and closure and the need for careful follow-up until completed healing is necessary is emphasised.

Tong et al. ${ }^{2}$ suggested that when extraction of maxillary posterior teeth was necessary, prophylactic antibiotics are not sufficient to prevent the complication of delayed healing. They suggested that the protocol of Marx for giving hyperbaric oxygen (HBO) should be considered even though they did not agree with wholesale prescription of HBO therapy.

So, if we were to base our surgery on these recommendations, we can safely perform lower third molar surgery with a prophylaxis antibiotic cover but an extraction of upper molar teeth (or even minor oral surgery of the upper third molar) would not benefit from it. I personally have had bad experiences with upper third molar extractions (even autoexfoliation) whereby prophylactic and/or therapeutic antibiotics have been of no advantage to the wound healing. As such, 
I advise my fellow colleagues to weigh carefully the need of prophylactic antibiotics in patients with a history of radiotherapy.

\section{W. C. Ngeow}

Malaysia

doi: 10.1038/sj.bdj.4812446

1. Kanatas A N, Rogers S N, Martin M V. A survey of antibiotic prescribing by maxillofacial consultants for dental extractions following radiotherapy to the oral cavity. Br Dent J 2002; 192: 157-160.

2. Tong A C, Leung A C, Cheng J C, Sham J. Incidence of complicated healing and osteoradionecrosis following tooth extraction in patients receiving radiotherapy for treatment of nasopharyngeal carcinoma. Aust DentJ 1999; 44: 187-194.

\section{Patient perception}

Sir, I read with interest B.Littler's letter 'Phantom Bite revisited' (BDJ 2005, 198:149). Dr. Litter quite rightly states that we should focus on the cause, which is clenching and the lack of proprioceptive feedback.

A patient's perception of his bite is a result of the proprioception from his masticatory system. This involves receptors in the temporo mandibular joint, muscles of mastication and in the periodontal membrane. High filling, whiplash injury causing disc displacement in the temporo mandibular joint or trauma to any of the masticatory muscles can cause this. Usually the patient's ability to adapt resolves this matter, with or without a little help from his dentist. I have observed that the muscles of patients who have a parafunctional habit eg clenching/grinding of the teeth are "hypersensitive". These can eventually, though not always lead to symptoms of TMD. These are the patients who may develop the phantom bite. The initiating cause may have been a visit to the dentist. Even keeping the mouth open for a long time can cause the "hypersensitive" muscles to go beyond the adaptability of the patient, and the phantom bite develops. If we can relax these sensitive muscles, the patient will start to accept his bite. Two case studies illustrate this phenomenon.

Patient A had a full upper denture made and found that when she wore this denture it caused her considerable facial pain and could not wear it for more than 30 minutes. Examination showed that the vertical dimension had been opened $7 \mathrm{~mm}$ beyond the resting vertical position. All the muscles of mastication were extremely tender.

Although the increased vertical dimension had improved the face aesthetically according to the Golden Proportion Rule, she felt that her bite wasn't right and her teeth didn't meet properly and this was causing all the facial pain.

I made her a pivot appliance causing the vertical to open by another $5 \mathrm{~mm}$. The patient was made aware of her clenching habits and taught by biofeedback to maintain a freeway space at all times. Within a month there was a dramatic improvement in her muscles. The patient found it difficult to be without her upper denture, and now no longer needs to wear the pivot appliance and her bite is no longer a problem.

Patient B, a patient with full dentition came in complaining of his bite being wrong and having difficulty in chewing and constantly biting his cheek and tongue.

Examination showed decreased lower facial height and very tender muscles of mastication. A pivot appliance was made, which initially made the symptoms worse, but gradually by controlling the clenching with biofeedback, and carefully monitoring the use of the pivot appliance, there was an improvement in the muscles of mastication. The patient no longer feels that his bite is wrong, his muscles look much more relaxed and his facial features look aesthetically more pleasing.

I hope these two cases illustrate the fact that we shouldn't readily refer patients for psychiatric treatment until all other methods have been tried. Biofeedback and pivot appliance give very good results.

N. K. Mohindra

By email

doi: 10.1038/sj.bdj.4812447

\section{Guest Leaders}

Guest leaders in the $B D J$ are there to provide an opportunity for anyone involved in dentistry (including patients) to write an appropriate comment for publication. These are published to accompany the usual Leader from the Editor-in-Chief.

Submissions must be between 200 and 500 words, typed and double-spaced. Name, address and telephone number should be supplied, as well as your position in the dental world.

For further help and guidance, please contact:

The Editor-in-Chief, British Dental Journal, 64 Wimpole Street, London W1G 8YS

E-mail:k.maynard@nature.com 